\title{
Dendritic Cell-targeting Lentiviral Vector ID-LV305
}

National Cancer Institute

\section{Source}

National Cancer Institute. Dendritic Cell-targeting Lentiviral Vector ID-LV305. NCI

Thesaurus. Code C115976.

An eng ineered lentiviral vector targeting dendritic cells (DCs) and containing nucleic acids encoding for the human tumor-associated cancer-testis antigen NY-ESO-1, with potential immunostimulatory and antineoplastic activities. Upon intradermal administration, the DC-targeting lentiviral vector ID-LV305 targets and binds to dermal DCs via the DC-specific intercellular adhesion molecule-3-grabbing non-integrin (DCSIGN) receptor. Upon internalization of the vector, the NY-ESO-1 protein is expressed, stimulates DC maturation and activates the immune system to mount a cytotoxic Tlymphocyte $(C T L)$ response against NY-ESO-1-expressing cells, which may result in tumor cell lysis. NY-ESO-1 is expressed in normal testes and on the surfaces of various tumor cells, and plays a key role in tumor cell proliferation and survival. 